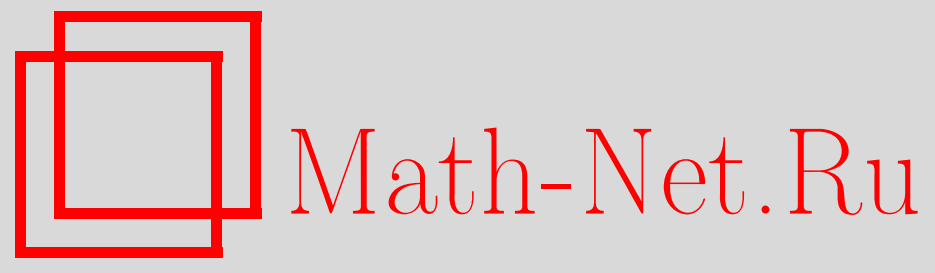

Д. А. Шляхин, О. В. Ратманова, Вынужденные осесимметричные колебания круглых многослойных биморфных пластин, Вестн. Сам. гос. техн. ун-та. Сер. Физ.-мат. науки, 2017, номер 4, 773785

DOI: https://doi.org/10.14498/vsgtu1564

Использование Общероссийского математического портала MathNet.Ru подразумевает, что вы прочитали и согласны с пользовательским соглашением

http://www . mathnet.ru/rus/agreement

Параметры загрузки:

IP: 54.224 .187 .69

26 апреля 2023 г., 18:12:20

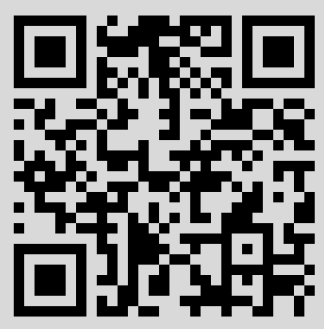


Вестн. Сам. гос. техн. ун-та. Сер. Физ.-мат. науки. 2017. Т. 21, № 4 . С. $773-785$ ISSN: 2310-7081 (online), 1991-8615 (print)

УДК 539.3

\title{
Вынужденные осесимметричные колебания круглых многослойных биморфных пластин
}

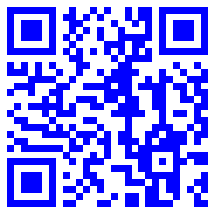

\section{Д. А. Шляхин, О. В. Ратманова}

Самарский государственный технический университет, Россия, 443100, Самара, ул. Молодогвардейская, 244.

\begin{abstract}
Аннотация
Представлена методика расчета круглых сплошных многослойных биморфных пластин и получены новые замкнутые решения осесимметричных динамических задач прямого и обратного пьезоэффектов. В общем случае при исследовании электроупругого (пьезокерамического) и упругого слоев математическая формулировка рассматриваемых задач включает уравнения движения и Максвелла в пространственной постановке относительно компонент вектора перемещений и потенциала электрического поля, а также соответствующие начально-краевые условия. Рассмотрены случаи шарнирного и жесткого закрепления внешнего контура конструкции. Для исследования связанных линейных задач применяется математический аппарат в виде метода конечных интегральных преобразований Фурье-Бесселя и обобщенного интегрального преобразования (КИП). При этом на каждом этапе исследования использовалась процедура приведения граничных условий к виду, позволяющему выполнять соответствующее преобразование.

Построенные расчетные соотношения позволяют обосновать конструктивные решения многослойных пьезокерамических преобразователей, а именно подобрать геометрические размеры и физические характеристики используемых материалов, определить размеры разрезных круговых электродов, позволяющие наиболее эффективно преобразовать внешнее электрическое воздействия в механические колебания при различной частоте. Кроме этого, появляется возможность проанализировать напряженно-деформированное состояние, характер изменения электрического поля, а также частотный спектр собственных осесимметричных колебаний рассматриваемых систем.
\end{abstract}

Ключевые слова: биморфная пластина, электроупругость, нестационарная нагрузка, интегральные преобразования.

\section{Краткое сообщение}

(2) (7) Контент публикуется на условиях лицензии Creative Commons Attribution 4.0 International (https://creativecommons.org/licenses/by/4.0/deed.ru)

\section{Образец для цитирования}

Шляхин Д. А., Ратманова О. В. Вынужденные осесимметричные колебания круглых многослойных биморфных пластин // Вестн. Сам. гос. техн. ун-та. Сер. Физ.-мат. науки, 2017. Т. 21, № 4. С. 773-785. doi: 10.14498/vsgtu1564.

\section{Сведения об авторах}

Дмитрий Аверкиевич Шляхин

доктор технических наук, доцент; заведующий кафедрой; каф. сопротивления материалов и строительной механики; e-mail: d-612-mit2009@yandex.ru

Олеся Викторовна Ратманова (D) http://orcid.org/0000-0001-7591-4921

ассистент; каф. сопротивления материалов и строительной механики;

e-mail: olesya654@yandex.ru 
Получение: 14 октября 2017 г. / Исправление: 9 декабря 2017 г. Принятие: 18 декабря 2017 г. / Публикация онлайн: 29 декабря 2017 г.

Введение. В различных электроакустических, оптических и пневматических приборах применяются пьезокерамические преобразователи в виде тонких биморфных пластин [1-3]. Данные конструкции представляют собой симметричные или асимметричные многослойные системы, состоящие из электроупругих и упругих тонких пластин. Их широкое применение объясняется высокими эксплуатационными параметрами, низкой стоимостью и простотой в управлении. Изгибные колебаний в рассматриваемых конструкциях создаются с помощью электрического напряжения, приложенного к электродированным поверхностям пьезокерамических пластин (явление обратного пьезоэффекта), или в случае действия механической нагрузки (прямой пьезоэффект).

Для описания работы и повышения функциональных возможностей биморфных пластин возникает необходимость в разработке математических моделей и построении общих решений, позволяющих описать сложную картину взаимодействия полей напряжений различной физической природы [4]. В настоящее время для реализации данной проблемы, как правило, применяются прикладные теории для тонких пластин в условиях установившегося режима вынужденных колебаний при использовании различных допущений о характере распределения напряженности электрического поля в пьезокерамических пластинах [5-11]. Полученные приближенные расчетные соотношения используются в дальнейшем для расчета и конструирования приборов различного назначения [12-14].

Замкнутые решения динамических начально-краевых задач электроупругости в пространственной постановке при аксиальной поляризации пьезокерамического материала получены в работах [15-17] только для однородного диска (цилиндра). В [15] рассматривался незакрепленный цилиндр при учете смешанных краевых условий на торцевых поверхностях, а в [16] исследовалось напряженно-деформированное состояние жестко закрепленной толстой пластины в случае действия осесимметричной механической нагрузки. Здесь также можно отметить одну из работ, посвященную определению спектра частот собственных осесимметричных и неосесимметричных колебаний [17] пьезокерамических цилиндров.

Таким образом, в настоящее время отсутствуют замкнутые решения динамических задач электроупругости для многослойных конструкций в пространственной постановке. Для решения этого вопроса в данной работе представлена методика расчета круглых многослойных шарнирно и жестко закрепленных биморфных сплошных пластин в случае действия электромеханической нагрузки. Построенные расчетные соотношения позволяют дать качественную и количественную оценку связанности электрических и механических полей напряжений в многослойных конструкциях. Частные решения данного подхода приведены в публикациях авторов [18,19].

1. Постановка задачи. В общем случае круглая биморфная конструкция, занимающая в цилиндрической системе координат область $\Omega=\{(r, \theta, z)$ : $\left.0 \leqslant r \leqslant b, 0 \leqslant \theta \leqslant 2 \pi, 0 \leqslant z \leqslant h^{*}\right\}$, представляет многослойную систему в ви- 
де жестко соединенных между собой электроупругих (пьезокерамических) и упругих тонких пластин. Для определенности принимаем, что она состоит из металлической подложки толщиной $h_{2}^{*}$ и двух пьезокерамических пластин высотой $h_{1}^{*}$, выполненных из материала гексагональной системы класса $6 \mathrm{~mm}$ $\left(h^{*}=2 h_{1}^{*}+h_{2}^{*}\right)$. Продольно-поперечные осесимметричные колебания индуцируются за счет подведения к торцевым электродированным поверхностям пьезопластин с различным направлением вектора аксиальной поляризации электрического напряжения $V^{*}\left(r_{*}, t_{*}\right)$ (рис. сверху) или действии на лицевых плоскостях конструкции механических нагрузки в виде нормальных напряжений $q^{*}\left(r_{*}, t_{*}\right)$ (рис. снизу). В дальнейшем все рассматриваемые величины будут обозначаться с символом *, а безразмерные - без символа. Рассматривается жесткое и шарнирное закрепления внешнего контура электроупругой системы (см. рис.).
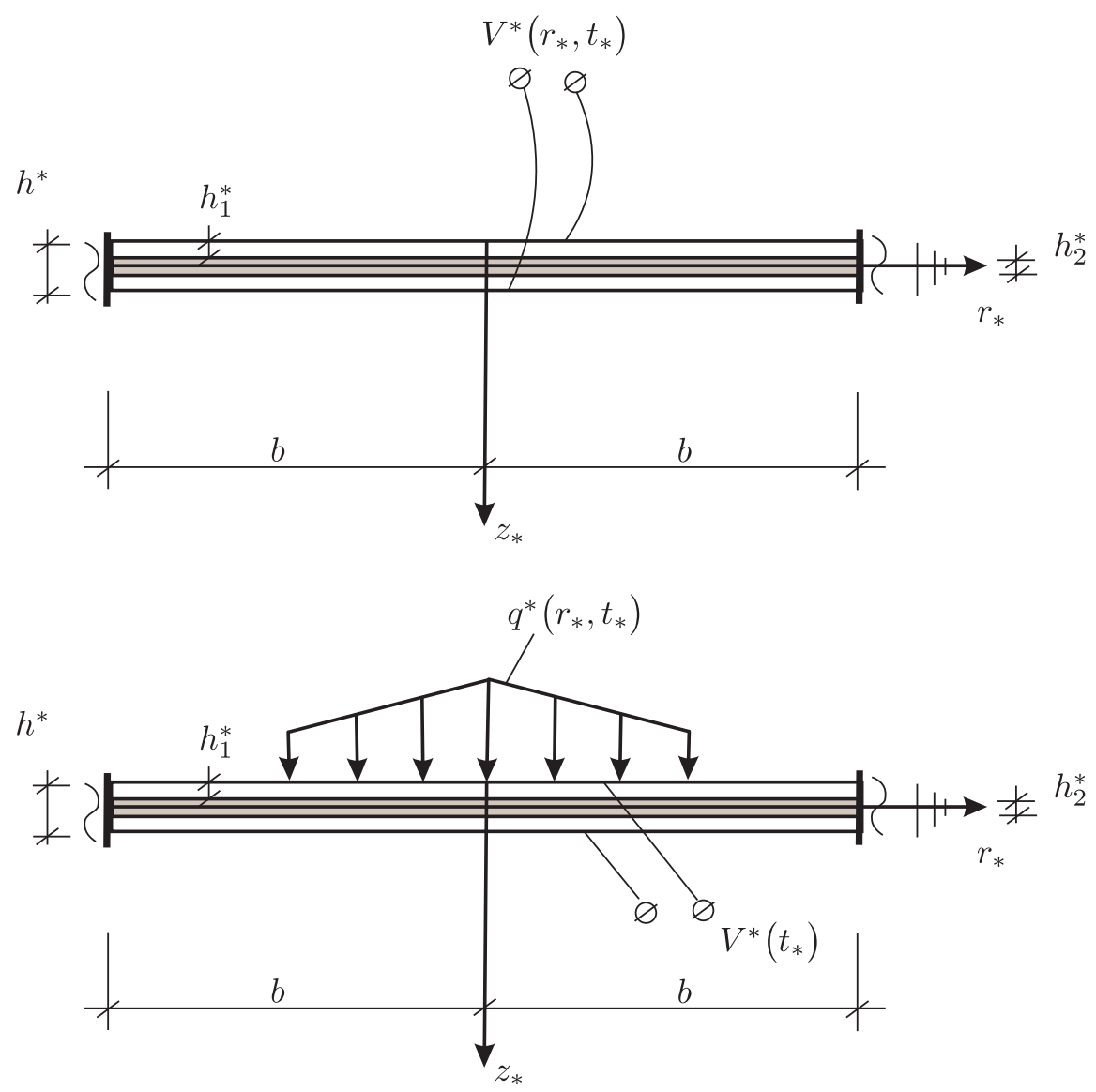

Расчетная схема биморфных пластин [Calculation scheme of bimorph plates]

Математическая формулировка рассматриваемых осесимметричных начально-краевых задач в безразмерной форме в цилиндрической системе координат включает следующие соотношения [4]:

- дифференциальные уравнения движения и Максвелла относительно безразмерных компонент вектора перемещений $U(r, z, t), W(r, z, t)$, а также 
безразмерного потенциала электрического поля $\varphi(r, z, t)$ :

$$
\begin{aligned}
& \frac{\partial}{\partial r} \nabla U+\frac{C_{55}^{(s)}}{C_{11}^{(s)}} \frac{\partial^{2} U}{\partial z^{2}}+\frac{C_{13}^{(s)}+C_{55}^{(s)}}{C_{11}^{(s)}} \frac{\partial^{2} W}{\partial r \partial z}+ \\
&+\frac{e_{31}+e_{15}}{e_{33}} \frac{\partial^{2} \varphi}{\partial r \partial z}-\Phi^{(s)} \frac{\partial^{2} U}{\partial t^{2}}=0 \\
& \frac{C_{55}^{(s)}}{C_{11}^{(s)}} \nabla \frac{\partial W}{\partial r}+\frac{C_{33}^{(s)}}{C_{11}^{(s)}} \frac{\partial^{2} W}{\partial z^{2}}+\frac{C_{13}^{(s)}+C_{55}^{(s)}}{C_{11}^{(s)}} \frac{\partial}{\partial z} \nabla U+ \\
&+\frac{e_{15}}{e_{33}} \nabla \frac{\partial \varphi}{\partial r}+\frac{\partial^{2} \varphi}{\partial z^{2}}-\Phi^{(s)} \frac{\partial^{2} W}{\partial t^{2}}=0 \\
& \frac{e_{31}+e_{15}}{e_{33}} \frac{\partial}{\partial z} \nabla U+\frac{e_{15}}{e_{33}} \nabla \frac{\partial W}{\partial r}+\frac{\partial^{2} W}{\partial z^{2}}- \\
&-\frac{C_{11}^{(1)} \varepsilon_{11}}{e_{33}^{2}} \nabla \frac{\partial \varphi}{\partial r}-\frac{C_{11}^{(1)} \varepsilon_{33}}{e_{33}^{2}} \frac{\partial^{2} \varphi}{\partial z^{2}}=0
\end{aligned}
$$

где

$$
\{U, W, r, z\}=\left\{U^{*}, W^{*}, r_{*}, z_{*}\right\} / b, \quad \varphi=\varphi^{*} e_{33} /\left(b C_{11}^{(1)}\right),
$$

$t=t_{*} b^{-1} \sqrt{C_{11}^{(1)} / \rho^{(1)}}-$ время; $U^{*}\left(r_{*}, z_{*}, t_{*}\right), W^{*}\left(r_{*}, z_{*}, t_{*}\right)-$ радиальная и аксиальная компоненты вектора перемещений; $\varphi^{*}\left(r_{*}, z_{*}, t_{*}\right)$ - потенциал электрического поля; $e_{m k}, \varepsilon_{11}, \varepsilon_{33}$ - пьезомодули и коэффициенты диэлектрической проницаемости электроупругого материала, $m, k=$ $=1,2, \ldots, 5 ; \rho^{(s)}, C_{m k}^{(s)}$ - объемная плотность и модули упругости электроупругого $(s=1)$ и упругого $(s=2)$ материалов; $\Phi^{(1)}=1, \Phi^{(2)}=$ $=\frac{C_{11}^{(2)} \rho^{(1)}}{C_{11}^{(1)} \rho^{(2)}}, \nabla=\frac{\partial}{\partial r}+\frac{1}{r}$

- механические граничные условия:

$$
|W(0, z, t)|<\infty, \quad|U(0, z, t)|<\infty
$$

жесткое закрепление:

$$
W(1, z, t)=0, \quad U(1, z, t)=0
$$

шарнирное закрепление:

$$
\begin{gathered}
W(1, z, t)=0 \\
\left.\sigma_{r r}\right|_{r=1}=\left[\frac{C_{11}^{(s)}}{C_{11}^{(1)}} \frac{\partial U}{\partial r}+\frac{C_{12}^{(s)}}{C_{11}^{(1)}} \frac{U}{r}+\frac{C_{13}^{(s)}}{C_{11}^{(1)}} \frac{\partial W}{\partial z}+\frac{e_{31}}{e_{33}} \frac{\partial \varphi}{\partial z}\right]_{r=1}=0 \\
\left.\sigma_{z z}\right|_{z=0}=\left[\frac{C_{13}^{(1)}}{C_{11}^{(1)}} \nabla U+\frac{C_{33}^{(1)}}{C_{11}^{(1)}} \frac{\partial W}{\partial z}+\frac{\partial \varphi}{\partial z}\right]_{z=0}=q \\
\left.\sigma_{r z}\right|_{z=0}=\left[\frac{C_{55}^{(1)}}{C_{11}^{(1)}}\left(\frac{\partial W}{\partial r}+\frac{\partial U}{\partial z}\right)+\frac{e_{15}}{e_{33}} \frac{\partial \varphi}{\partial r}\right]_{z=0}=0
\end{gathered}
$$




$$
\begin{gathered}
\left.\sigma_{z z}\right|_{z=h}=\left[\frac{C_{13}^{(1)}}{C_{11}^{(1)}} \nabla U+\frac{C_{33}^{(1)}}{C_{11}^{(1)}} \frac{\partial W}{\partial z}+\frac{\partial \varphi}{\partial z}\right]_{z=h}=0 \\
\left.\sigma_{r z}\right|_{z=h}=\left[\frac{C_{55}^{(1)}}{C_{11}^{(1)}}\left(\frac{\partial W}{\partial r}+\frac{\partial U}{\partial z}\right)+\frac{e_{15}}{e_{33}} \frac{\partial \varphi}{\partial r}\right]_{z=h}=0
\end{gathered}
$$

$$
\begin{gathered}
{\left[\frac{C_{55}^{(1)}}{C_{11}^{(1)}}\left(\frac{\partial W}{\partial r}+\frac{\partial U}{\partial z}\right)+\frac{e_{15}}{e_{33}} \frac{\partial \varphi}{\partial r}\right]_{z=h_{1}+h_{2}}=\left[\frac{C_{55}^{(2)}}{C_{11}^{(1)}}\left(\frac{\partial W}{\partial r}+\frac{\partial U}{\partial z}\right)\right]_{z=h_{1}+h_{2}}} \\
W(z+0)=W(z-0), \quad U(z+0)=U(z-0)
\end{gathered}
$$

- электрические граничные условия:

$$
\begin{aligned}
& \varphi(0, z, t)<\infty \\
& \left.D_{r}\right|_{r=1}=\left[-\frac{C_{11}^{(1)} \varepsilon_{11}}{e_{33}^{2}} \frac{\partial \varphi}{\partial r}+\frac{e_{15}}{e_{33}}\left(\frac{\partial W}{\partial r}+\frac{\partial U}{\partial z}\right)\right]_{r=1}=0
\end{aligned}
$$

а) задача обратного пьезоэффекта

$$
\begin{gathered}
\varphi(r, 0, t)=V(r, t) / 2, \quad \varphi(r, h, t)=-V(r, t) / 2, \\
\varphi\left(r, h_{1}, t\right)=\varphi\left(r, h_{1}+h_{2}, t\right)=0
\end{gathered}
$$

б) задача прямого пьезоэффекта

$$
\left.D_{z}\right|_{z=\left\{0, h_{1}, h_{1}+h_{2}, h\right\}}=\left[-\frac{C_{11}^{(1)} \varepsilon_{33}}{e_{33}^{2}} \frac{\partial \varphi}{\partial z}+\frac{e_{31}}{e_{33}} \nabla U+\frac{\partial W}{\partial z}\right]_{z=\left\{0, h_{1}, h_{1}+h_{2}, h\right\}}=0 ;
$$

- начальные условия:

$$
\begin{aligned}
& U(r, z, 0)=U_{0}(r, z),\left.\quad \frac{\partial U}{\partial t}\right|_{t=0}=\dot{U}_{0}(r, z), \\
& W(r, z, 0)=W_{0}(r, z),\left.\quad \frac{\partial W}{\partial t}\right|_{t=0}=\dot{W}_{0}(r, z),
\end{aligned}
$$

где $\left\{U_{0}, \dot{U}_{0}, W_{0}, \dot{W}_{0}, h, h_{1}, h_{2}\right\}=\left\{U_{0}^{*}, \dot{U}_{0}^{*}, W_{0}^{*}, \dot{W}_{0}^{*}, h^{*}, h_{1}^{*}, h_{2}^{*}\right\} / b ; \sigma_{p m}-$ компоненты тензора напряжений, $p, m=r, z ; D_{r}, D_{z}$ - компоненты вектора индукции электрического поля; $U_{0}^{*}, \dot{U}_{0}^{*}, W_{0}^{*}, \dot{W}_{0}^{*}$ - известные в начальный момент времени перемещения и их скорости; $q=q^{*} / C_{11}^{(1)}$; $V=V^{*} e_{33} /\left(b C_{11}^{(1)}\right)$.

При исследовании упругой среды $(s=1)$ система (1) состоит только из уравнений движения, сформулированных относительно компонент вектора перемещений. Выражения (2) и первое неравенство (8) являются условиями ограниченности решения в центре пластины, равенство $\left.D_{r}\right|_{r=1}=0$ (8) 
учитывает отсутствие электродного покрытия на цилиндрической поверхности пластины, а выражения (10) в задаче прямого пьезоэффекта означают подключение электродированной поверхности пьезокерамической пластины к измерительному прибору с большим входным сопротивлением, что соответствует режиму «холостого хода» (отсутствию свободных электрических зарядов). Формулы (7) позволяют учесть условия совместности напряжений и перемещений в плоскости жесткого соединения пластин. Кроме этого, в задаче обратного пьезоэффекта первое равенство (5) приравнивается к нулю.

2. Построение решения. Определение общего интеграла рассматриваемых краевых задач электроупругости выполняется путем последовательного применения конечных интегральных преобразований: Фурье-Бесселя [20] по радиальной переменной и обобщенного преобразования [21] по аксиальной координате. При этом на каждом этапе предварительно выполняется процедура приведения соответствующих граничных условий к виду, позволяющему использовать алгоритм преобразования.

Преобразование Фурье--Бесселя [20] позволяет удовлетворить только смешанные граничные условия. Для выполнения данного требования необходимо произвести следующие замены граничных условий (3), (4):

- при жестком защемлении пластины первое равенство (3) заменяется условием наличия касательных напряжений $N_{1}(z, t)$ на цилиндрической поверхности пластины:

$$
\left.\sigma_{r z}\right|_{r=1}=\left[\frac{C_{55}}{C_{11}}\left(\frac{\partial W}{\partial r}+\frac{\partial U}{\partial z}\right)+\frac{e_{15}}{e_{33}} \frac{\partial \varphi}{\partial r}\right]=N_{1}(z, t) ;
$$

- в случае шарнирного закрепления последнее соотношение (8) заменяется условием наличия потенциала электрического поля $\varphi_{1}(z, t)$ на цилиндрической поверхности при $r=1$ :

$$
\varphi(1, z, t)=\varphi_{1}(z, t)
$$

Кроме этого, в общем случае вводятся новые функции $u(r, z, t), w(r, z, t)$, $\phi(r, z, t)$, связанные с $U(r, z, t), W(r, z, t), \varphi(r, z, t)$ соотношениями

$$
\begin{gathered}
U(r, z, t)=-\frac{e_{31}}{2 e_{33}} \frac{\partial \varphi_{1}(z, t)}{\partial z} r\left[H\left(z-h_{1}\right)+H\left(z-h_{1}-h_{2}\right)\right]+u(r, z, t), \\
W(r, z, t)=W_{1}(t)+A_{0} C_{11} \varepsilon_{11} r^{2} N_{1}(z, t)+w(r, z, t), \\
\varphi(r, z, t)=A_{0} e_{33} e_{15} r^{2} N_{1}(z, t)+\phi(r, z, t), \quad A_{0}=\left[2\left(C_{55} \varepsilon_{11}+e_{15}^{2}\right)\right]^{-1} .
\end{gathered}
$$

Здесь $H(\cdot)$ - единичная функция Хэвисайда [22]; $W_{1}(t), N_{1}(z, t), \varphi_{1}(z, t)-$ неизвестные функции, определяемые в процессе решения задачи, с помощью которых удается удовлетворить все условия (3), (4), (8).

В результате подстановки (14) в (1)-(13) получаем новую краевую задачу относительно функций $u, w, \phi$. При этом дифференциальные уравнения (1), граничные условия по переменной $z(5)-(10)$ становятся неоднородными с правыми частям $R_{1}, R_{2}, R_{3}, B_{1}, B_{2}, \ldots, B_{10}$, начальные условия $U_{0}, \dot{U}_{0}, W_{0}$, $\dot{W}_{0}$ следует заменить на $u_{0}, \dot{u}_{0}, w_{0}, \dot{w}_{0}$, а краевые условия (2)-(4) принимают следующий вид: 
- жесткое закрепление:

$$
U(1, z, t)=0,\left.\quad \frac{\partial W}{\partial r}\right|_{z=1}=0,\left.\quad \frac{\partial \phi}{\partial r}\right|_{z=1}=0
$$

- шарнирное закрепление:

$$
W(1, z, t)=0, \quad \phi(1, z, t)=0,\left.\quad \nabla U\right|_{r=1}=0
$$

Последнее условие (15) получается при $C_{11}^{(s)}=C_{12}^{(s)}$.

Применим к краевой задаче относительно функций $u, w, \phi$ конечные интегральные преобразования Фурье-Бесселя, используя следующие трансформанты:

$$
\begin{gathered}
u_{H}\left(j_{n}, z, t\right)=\int_{0}^{1} u(r, z, t) r J_{1}\left(j_{n} r\right) d r \\
\left\{w_{H}\left(j_{n}, z, t\right), \phi_{H}\left(j_{n}, z, t\right)\right\}=\int_{0}^{1}\{w(r, z, t), \phi(r, z, t)\} r J_{0}\left(j_{n} r\right) d r
\end{gathered}
$$

и формулы обращения:

$$
\begin{gathered}
u(r, z, t)=2 \sum_{n=1}^{\infty} \frac{u_{H}\left(j_{n}, z, t\right)}{S\left(j_{n}\right)^{2}} J_{1}\left(j_{n} r\right), \\
\{w(r, z, t), \phi(r, z, t)\}=2 \sum_{n=0}^{\infty} \frac{\left\{w_{H}\left(j_{n}, z, t\right), \phi_{H}\left(j_{n}, z, t\right)\right\}}{S\left(j_{n}\right)^{2}} J_{0}\left(j_{n} r\right),
\end{gathered}
$$

где $j_{n}$ - положительные нули функций $J_{1}\left(j_{n}\right) ; J_{0}\left(j_{n}\right)$ соответственно при жестком и шарнирном закреплении пластины, расположенные в порядке их возрастания, $n=0,1,2, \ldots, j_{0}=0 ; S\left(j_{n}\right)=J_{0}\left(j_{n}\right)$ при жестком и $S\left(j_{n}\right)=J_{1}\left(j_{n}\right)$ при шарнирном закреплении.

В результате получаем новую задачу относительно трансформант Фурье-Бесселя $u_{H}, w_{H}, \phi_{H}$, которую решаем, используя обобщенный метод конечных интегральных преобразований [21] по аксиальной координате. При этом предварительно проводится также процедура стандартизации, связанная с приведением неоднородных граничных условий по переменной $z$ к однородным. Для этой цели вводятся новые функции $U_{H}, W_{H}, \varphi_{H}$, связанные с $u_{H}, w_{H}, \phi_{H}$ соотношениями

$$
\begin{aligned}
\left\{u_{H}\left(j_{n} z, t\right), w_{H}\left(j_{n} z, t\right), \phi_{H}\left(j_{n} z, t\right)\right\} & = \\
=\left\{P_{1}\left(j_{n} z, t\right)\right. & \left., P_{2}\left(j_{n} z, t\right), P_{3}\left(j_{n} z, t\right)\right\}+ \\
& +\left\{U_{H}\left(j_{n} z, t\right), W_{H}\left(j_{n} z, t\right), \varphi_{H}\left(j_{n} z, t\right)\right\} .
\end{aligned}
$$

В результате подстановки (17) в расчетные соотношения краевой задачи относительно $u_{H}, w_{H}, \phi_{H}$ получаем новую задачу для функций $U_{H}, W_{H}$, $\varphi_{H}$. При этом граничные условия с помощью выбора функций $P_{1}, P_{2}, P_{3}$ становятся однородными.

Начально-краевую задачу относительно функций $U_{H}, W_{H}, \varphi_{H}$ решаем, используя обобщенный метод конечных интегральных преобразований (КИП) 
[21] с использованием неизвестной трансформанты $G\left(\lambda_{i n}, n, t\right)$ и компонент $K_{1}\left(\lambda_{i n}, z\right), K_{2}\left(\lambda_{i n}, z\right), K_{3}\left(\lambda_{i n}, z\right)$ вектор-функции ядра преобразования $\left(\lambda_{i n}-\right.$ положительные параметры, образующие счетное множество, $i=1,2,3, \ldots$ )

$$
G\left(\lambda_{i n}, n, t\right)=\int_{0}^{h}\left(U_{H} K_{1 i n}+W_{H} K_{2 i n}\right) d z,
$$

и формул обращения

$$
\begin{aligned}
\left\{U_{H}, W_{H}, \varphi_{H}\right\} & =\sum_{i=1}^{\infty} G_{i n}\left\{K_{1 i n}, K_{2 i n}, K_{3 i n}\right\}\left\|K_{i n}\right\|^{-2} \\
\left\|K_{i n}\right\|^{2} & =\int_{0}^{h}\left(K_{1 i n}^{2}+K_{2 i n}^{2}+K_{3 i n}^{2}\right) d z
\end{aligned}
$$

Общие решения системы дифференциальных уравнений для электроупругого $(s=1)$ и упругого $(s=2)$ слоев представлены в работах авторов [16,23].

Подстановка расчетных соотношений $K_{1}\left(\lambda_{i n}, z\right), K_{2}\left(\lambda_{i n}, z\right), K_{3}\left(\lambda_{i n}, z\right)$ в граничные условия по аксиальной координате формирует однородную систему уравнений относительно постоянных интегрирования $D_{1 i n}, D_{2 i n}, \ldots, D_{16 i n}$. Разыскивая ее нетривиальное решение, получаем трансцендентное уравнение для вычисления собственных значений $\lambda_{i n}$, а также выражения для постоянных $D_{1 i n}, D_{2 i n}, \ldots, D_{16 i n}$.

Окончательные выражения функций $U(r, z, t), W(r, z, t), \varphi(r, z, t)$ получим, применяя к трансформанте (18) последовательно формулы обращения КИП (19) и Фурье-Бесселя (16). В результате с учетом (14), (17) имеем

$$
\begin{aligned}
U(r, z, t)=-\frac{e_{31}}{2 e_{33}} \frac{\partial \varphi_{1}(z, t)}{\partial z} r & {\left[H\left(z-h_{1}\right)+H\left(z-h_{1}-h_{2}\right)\right]+} \\
+ & 2 \sum_{n=1}^{\infty} \frac{J_{1}\left(j_{n} r\right)}{S\left(j_{n}\right)^{2}}\left(P_{1}+\sum_{i=1}^{\infty} G_{i n} K_{1 i n}\left\|K_{i n}\right\|^{-2}\right),
\end{aligned}
$$

$W(r, z, t)=W_{1}(t)+A_{0} C_{11} \varepsilon_{11} r^{2} N_{1}(z, t)+$

$$
+2 \sum_{n=0}^{\infty} \frac{J_{0}\left(j_{n} r\right)}{S\left(j_{n}\right)^{2}}\left(P_{2}+\sum_{i=1}^{\infty} G_{i n} K_{2 i n}\left\|K_{i n}\right\|^{-2}\right)
$$

$$
\begin{aligned}
\varphi(r, z, t)=A_{0} e_{33} e_{15} r^{2} N_{1}(z, t) & + \\
& +2 \sum_{n=0}^{\infty} \frac{J_{0}\left(j_{n} r\right)}{S\left(j_{n}\right)^{2}}\left(P_{3}+\sum_{i=1}^{\infty} G_{i n} K_{3 i n}\left\|K_{i n}\right\|^{-2}\right) .
\end{aligned}
$$

Заключительным этапом исследования является определение зависимостей $W_{1}(t), N_{1}(z, t), \varphi_{1}(z, t)$. При решении задачи обратного пьезоэффекта первоначально решается задача в случае действия только электрической нагрузки $V(r, t)\left(N_{1}=0\right)$. 
В результате определяется функция $W_{1}(t)$ из условия отсутствия вертикальных перемещений цилиндрической поверхности пластины при $z=h / 2$, а также характер изменения касательных напряжений $\sigma_{r z}(1, z, t)$, который определяет в дальнейшем выражение для $N_{1}(z, t)$. На втором этапе решения рассматривается действие функции $N_{1}(z, t)$, которая аппроксимируется параболической зависимостью и определяется из условия равенства нулю перемещений на цилиндрической поверхности пластины в случае действия $V(r, t)$ и $N_{1}(z, t)$. Суммирование двух расчетов позволяет получить окончательное решение.

Аналогичным образом решается задача прямого пьезоэффекта. Первоначально рассматривается действие механической нагрузки $q(r, t)$, а на следующем этапе функция $\varphi_{1}(r, t)$ определяется из условия равенства нулю радиальной компоненты вектора индукции электрического поля на цилиндрической поверхности при суммировании двух расчетов.

Полученные расчетные соотношения (20) удовлетворяют дифференциальные уравнения (1) и краевые условия (2)-(11), т. е. представляют замкнутые решения рассматриваемых начально-краевых задач электроупругости для биморфных пьезокерамических пластин.

Заключение. В настоящей работе на основании разработанного в работах авторов $[16,18,19]$ алгоритма получены расчетные соотношения для решения частных задач. Представленная методика расчета позволяет получать замкнутые решения связанных осесимметричных нестационарных задач в пространственной постановке для круглых сплошных биморфных пластин на шарнирном и жестком закреплении по контуру.

Допущение об установившемся режиме вынужденных колебаний, используемое при исследовании динамических задач, справедливо только в случае, когда частоты внешнего гармонического воздействия существенно меньше первой частоты собственных колебаний.

Полученные расчетные соотношения позволяют определить напряженнодеформированное состояние, спектр частот собственных колебаний и характер распределения электрического поля в многослойных пьезокерамических преобразователях резонансного и нерезонансного классов в случае действия нестационарной нагрузки.

Конкурирующие интересы. Заявляем, что в отношении авторства и публикации этой статьи конфликта интересов не имеем.

Авторский вклад и ответственность. Все авторы принимали участие в разработке концепции статьи и в написании рукописи. Авторы несут полную ответственность за предоставление окончательной рукописи в печать. Окончательная версия рукописи была одобрена всеми авторами.

Финансирование. Исследование выполнялось без финансирования.

\section{Библиографический список}

1. Sharapov V. Bimorph and trimorph piezoelements/ Piezoceramic Sensors. Microtechnology and MEMS. Berlin, Heidelberg: Springer, 2011. pp. 179-229. doi:10.1007/ 978-3-642-15311-2_6.

2. Sharapov V., Sotula Z., Kunickaya L. Devices to control and diagnose bimorph piezoelements / Piezo-Electric Electro-Acoustic Transducers. Microtechnology and MEMS. Cham: Springer, 2013. pp. 191-212. doi: 10.1007/978-3-319-01198-1_11.

3. Seeley C. E., Delgado E., Kunzman J., Bellamy D. Miniature piezo composite bimorph actuator for elevated temperature operation / International Mechanical Engineering Congress 
and Exposition. vol. 10 (Seattle, Washington, USA, November 11-15, 2007), Mechanics of Solids and Structures, Parts A and B, 2007. pp. 405-415. doi: 10.1115/imece2007-44088.

4. Гринченко В. Т., Улитко А. Ф., Шульга Н. А. Механика связанных полей в элементах конструкиий. Киев: Наук. думка, 1989. 279 с.

5. Smits J. G., Dalke S. I., Cooney T. K. The constituent equations of piezoelectric bimorphs // Sensors and Actuators A: Physical, 1991. vol.28, no.1. pp. 41-61. doi: 10.1016/ 0924-4247 (91) 80007-C.

6. Ватульян А. О., Рынкова А. А. Об одной модели изгибных колебаний пьезоэлектрических биморфов с разрезными электродами и ее приложениях // Изв. PAН. MTT, 2007. № 4. C. 114-122.

7. Tsaplev V., Konovalov R., Abbakumov K. Disk bimorph-type piezoelectric energy harvester // Journal of Power and Energy Engineering, 2015. vol. 3, no. 4. pp. 63-68. doi: 10. 4236/jpee.2015.34010.

8. Цаплев В. М., Коновалов Р. С. Частотные зависимости констант высших порядков пьезоэлектрической керамики // Дефектоскопия, 2017. № 7. С. 14-22.

9. Янчевский И. В. Минимизация прогибов электроупругой биморфной пластины при импульсном нагружении // Проблемы вычислительной механики и прочности конструкu, иน, 2011. № 16 .

10. Шляхин Д. А. Вынужденные осесимметричные колебания пьезокерамической тонкой биморфной пластины // Изв. РАН. МТТ, 2013. № 2. С. 77-85.

11. Rashidifar M. A., Rashidifar A. A. Vibrations analysis of circular plate with piezoelectric actuator using thin plate theory and Bessel function // American Journal of Engineering, Technology and Society, 2015. vol. 2, no. 6. pp. 140-156.

12. Jam J. E., Khosravi M., Namdara N. An exact solution of mechanical buckling for functionally graded material bimorph circular plates // Metall. Mater. Eng., 2013. vol. 19, no. 1. pp. $45-63$.

13. Jandaghiana A. A., Jafarib A. A., Rahmania O. Vibrational response of functionally graded circular plate integrated with piezoelectric layers: An exact solution // Engineering Solid Mechanics, 2014. vol. 2, no. 2. pp. 120-130. doi: 10.5267/j.esm.2014.1.004.

14. Jurenas V., Bansevicius R., Navickaite S. Piezoelectric bimorphs for laser shutter systems: optimization of dynamic characteristics // Mechanika, 2010. no. 5(85). pp. 44-47.

15. Сеницкий Ю. Э., Шляхин Д. А. Нестационарная осесимметричная задача электроупругости для толстой круглой анизотропной пьезокерамической пластины // Изв. PAH. MTT, 1999. № 1. С. 78-87.

16. Шляхин Д. А. Вынужденные осесимметричные колебания толстой круглой жестко закрепленной пьезокерамической пластины // Изв. РАН. МTT, 2014. № 4. С. 90-100.

17. Шульга Н. А., Болкисев А. М. Колебания пъезоэлектрических тел. Киев: Наук. думка, 1990. 228 c.

18. Shlyakhin D. A., Kazakova O. V. A dynamic axially symmetric goal and its extended solution for a fixed rigid circular multi-layer plate// Procedia Engineering, 2016. vol. 153. pp. 662-666. doi: 10.1016/j.proeng. 2016.08.219.

19. Шляхин Д. А. Динамическая осесимметричная задача прямого пьезоэффекта для круглой биморфной пластины // Вестник Пермского начионального исследовательского политехнического университета. Механика, 2017. №1. С. 164-180. doi: 10.15593/ perm.mech/2017.1.10.

20. Sneddon I. N. Fourier Transforms. New York: McGraw-Hill Book Company, Inc., 1950.

21. Сеницкий Ю. Э. Исследование упругого деформирования элементов конструкиий при динамических воздействиях методом конечных интегральных преобразований. Саратов: Сарат. ун-т, 1985. $174 \mathrm{c.}$

22. Владимиров В. С. Обобщенные функиии в математической физике. М.: Наука, 1978. 318 с.

23. Шляхин Д. А. Вынужденные осесимметричные изгибные колебания толстой круглой жестко закрепленной пластины // Вестн. СамГУ. Естественнонаучн. сер., 2011. № $8(89)$. C. $142-152$. 


\title{
MSC: $74 \mathrm{~F}, 74 \mathrm{~K} 20$
}

\section{Forced axisymmetric oscillations of circular multilayer bimorph plates}

\section{A. Shlyakhin, O. V. Ratmanova}

Samara State Technical University,

244, Molodogvardeyskaya st., Samara, 443100, Russian Federation.

\begin{abstract}
A method for calculating circular multilayer bimorph plates is presented and new analytical solutions of axisymmetric dynamic problems of direct and inverse piezoelectric effects are obtained. The cases of hinged and rigid fixing of the outer contour of the structure are considered. To investigate related linear problems, a mathematical apparatus is used in the form of a method of finite integral transformations. The constructed calculated relationships allow us to substantiate the constructive solutions of piezoceramic transducers.

Built design ratio allows to prove the constructive solutions of multilayer piezoelectric ceramic transducers, namely, to choose the geometrical dimensions and physical characteristics of the materials used, define the dimensions of a split circular electrode, allowing most effectively to convert the external electrical stimulation into mechanical vibrations at various frequency. In addition, it is possible to perform stress-strain state, the nature of the change of the electric field and frequency range of the axisymmetric vibrations of the considered systems.
\end{abstract}

Keywords: bimorph plate, electroelasticity, nonstationary loading, integral transformations.

Received: $14^{\text {th }}$ October, $2017 /$ Revised: $9^{\text {th }}$ December, $2017 /$

Accepted: $18^{\text {th }}$ December, $2017 /$ First online: $29^{\text {th }}$ December, 2017

Competing interests. We declare that we have no conflicts of interests with the authorship and publication of this article.

Authors' contributions and responsibilities. Each author has participated in the article concept development and in the manuscript writing. The authors are absolutely

\section{Short Communication}

() () (i) The content is published under the terms of the Creative Commons Attribution 4.0 International License (http://creativecommons.org/licenses/by/4.0/)

Please cite this article in press as:

$\mathrm{S}$ hly ak h in D. A., R a t m a nova O. V. Forced axisymmetric oscillations of circular multilayer bimorph plates, Vestn. Samar. Gos. Tekhn. Univ., Ser. Fiz.-Mat. Nauki [J. Samara State Tech. Univ., Ser. Phys. Math. Sci.], 2017, vol. 21, no. 4, pp. 773-785. doi: 10.14498/vsgtu1564 (In Russian).

\section{Authors' Details:}

Dmitry A. Shlyakhin (10) http://orcid.org/0000-0003-0926-7388

Dr. Techn. Sci.; Head of Dept.; Dept. of Strength of Materials and Structural Mechanics; e-mail: d-612-mit2009@yandex.ru

Olesya V. Ratmanova (1) http://orcid.org/0000-0001-7591-4921

Assistant; Dept. of Strength of Materials and Structural Mechanics;

e-mail: olesya654@yandex.ru 
responsible for submitting the final manuscript in print. Each author has approved the final version of manuscript.

Funding. The research has not had any funding.

\section{References}

1. Sharapov V. Bimorph and trimorph piezoelements, In: Piezoceramic Sensors. Microtechnology and MEMS. Berlin, Heidelberg, Springer, 2011, pp. 179-229. doi:10.1007/ 978-3-642-15311-2_6.

2. Sharapov V., Sotula Z., Kunickaya L. Devices to control and diagnose bimorph piezoelements, In: Piezo-Electric Electro-Acoustic Transducers. Microtechnology and MEMS. Cham, Springer, 2013, pp. 191-212. doi: 10.1007/978-3-319-01198-1_11.

3. Seeley C. E., Delgado E., Kunzman J., Bellamy D. Miniature piezo composite bimorph actuator for elevated temperature operation, In: International Mechanical Engineering Congress and Exposition, vol. 10 (Seattle, Washington, USA, November 11-15, 2007), Mechanics of Solids and Structures, Parts A and B, 2007, pp. 405-415. doi: 10.1115/imece2007-44088.

4. Grinchenko V. T., Ulitko A. F., Shul'ga N. A. Mekhanika sviazannykh polei v elementakh konstruktsii [Mechanics of coupled fields in structural elements]. Kiev, Nauk. dumka, 1989, 279 pp. (In Russian)

5. Smits J. G., Dalke S. I., Cooney T. K. The constituent equations of piezoelectric bimorphs, Sensors and Actuators A: Physical, 1991, vol.28, no. 1, pp. 41-61. doi: 10.1016/ 0924-4247 (91) 80007-C.

6. Vatul'yan A. O., Rynkova A. A A model of bending vibrations of piezoelectric bimorphs with split electrodes and its applications, Mech. Solids, 2007, vol.42, no.4, pp. 595-602. doi : 10.3103/S0025654407040127.

7. Tsaplev V., Konovalov R., Abbakumov K. Disk bimorph-type piezoelectric energy harvester, Journal of Power and Energy Engineering, 2015, vol. 3, no. 4, pp. 63-68. doi : 10.4236/jpee. 2015.34010.

8. Tsaplev V. M., Konovalov R. S. Frequency dependences of higher-order constants of piezoelectric ceramics, Russ. J. Nondestruct. Test, 2017, vol.53, no.7, pp. 485-492. doi: 10. 1134/S1061830917070087.

9. Yanchevskiy I. V. Minimizing deflections of round electroelastic bimorph plate under impulsive loading, Problems of computational mechanics and strength of structures, 2011, no. 16 (In Russian).

10. Shlyakhin D. A. Forced nonstationary axisymmetric vibrations of a piezoceramic thin bimorph plate, Mech. Solids, 2013, vol.48, no.2, pp. 178-185. doi:10.3103/ S002565441302009X.

11. Rashidifar M. A., Rashidifar A. A. Vibrations analysis of circular plate with piezoelectric actuator using thin plate theory and Bessel function, American Journal of Engineering, Technology and Society, 2015, vol. 2, no. 6, pp. 140-156.

12. Jam J. E., Khosravi M., Namdara N. An exact solution of mechanical buckling for functionally graded material bimorph circular plates, Metall. Mater. Eng., 2013, vol. 19, no. 1, pp. 45-63.

13. Jandaghiana A. A., Jafarib A. A., Rahmania O. Vibrational response of functionally graded circular plate integrated with piezoelectric layers: An exact solution, Engineering Solid Mechanics, 2014, vol.2, no.2, pp. 120-130. doi: 10.5267/j.esm.2014.1.004.

14. Jurenas V., Bansevicius R., Navickaite S. Piezoelectric bimorphs for laser shutter systems: optimization of dynamic characteristics, Mechanika, 2010, no. 5(85), pp. 44-47.

15. Senitskii Yu. E., Shlyakhin D. A. The nonstationary axisymmetric problem of electroelasticity for a thick circular anisotropic piezoceramic plate, Mech. Solids, 1999, vol. 34, no. 1, pp. 66-74.

16. Shlyakhin D. A. Forced axisymmetric vibrations of a thick circular rigidly fixed piezoceramic plate, Mech. Solids, 2014, vol.49, no. 4, pp. 435-444. doi: 10.3103/S0025654414040086. 
17. Shul'ga N. A., Bolkisev A. M. Kolebaniia p'ezoelektricheskikh tel [Fluctuations of Piezoelectric Bodies]. Kiev, Nauk. dumka, 1990, 228 pp. (In Russian)

18. Shlyakhin D. A., Kazakova O. V. A dynamic axially symmetric goal and its extended solution for a fixed rigid circular multi-layer plate, Procedia Engineering, 2016, vol. 153, pp. 662666. doi : 10.1016/j.proeng.2016.08.219.

19. Shlyakhin D. A. Dynamic axisymmetric problem direct piezoeffect for round bimorph plate, PNRPU Mechanics Bulletin, 2017, no.1, pp. 164-180 (In Russian). doi: 10.15593/perm. mech/2017.1.10.

20. Sneddon I. N. Fourier Transforms. New York, McGraw-Hill Book Company, Inc., 1950.

21. Senitskii Yu. E. Issledovanie uprugogo deformirovaniia elementov konstruktsii pri dinamicheskikh vozdeistviiakh metodom konechnykh integral'nykh preobrazovanii [Investigation of the Elastic Deformation of Construction Elements under Dynamic Actions by the Method of Finite Integral Transforms]. Saratov, Saratov Univ., 1985, 174 pp. (In Russian)

22. Vladimirov V. S. Obobshchennye funktsii v matematicheskoi fizike [Generalized Functions in Mathematical Physics]. Moscow, Nauka, 1978, 318 pp. (In Russian)

23. Shlyakhin D. A. The compelled axisymmetric bending fluctuations of thick round rigid plate, Vestnik Samarskogo Gosudarstvennogo Universiteta. Estestvenno-Nauchnaya Seriya, 2011, no. 8(89), pp. 142-152 (In Russian). 